\title{
DLR High altitude balloon launched experimental glider (HABLEG): system design, control and flight data analysis
}

\author{
Maximilian Laiacker, Sven Wlach and Marc Schwarzbach
}

\begin{abstract}
In this paper the autopilot setup and the lessons learned from a successful balloon launched high altitude glider mission will be presented. Flying an unmanned aerial vehicle inside the stratosphere means operating it outside visual line of sight, in low pressures and cold temperatures. These environmental conditions pose many challenges to the UAV aerodynamic design, the autopilot system and the ground infrastructure. In May 2015 the HABLEG airplane was carried up to an altitude of $20 \mathrm{~km}$ by a balloon. When the target altitude was reached it automatically transitioned to horizontal flight and landed back at the landing site where the balloon was released 145 minutes earlier. We will present and analyzed flight test result from every phase of the mission and summarize the lessons learned.
\end{abstract}

\section{INTRODUCTION}

Unmanned flying in the higher atmosphere has come into focus in recent years. After decades of manned high altitude flying [X15, U2, SR71], key technology evolved in the 1990's which mainly lead to the NASA family of high altitude solar planes for example Pathfinder and Helios. As a result of ongoing work, a flight of two weeks could be achieved by the Qinetic Zephyr airplane in 2010, which is the reference to this date. Flying at high altitude is necessary for these missions to stay above the clouds. Flying below the clouds would prevent the plane from collecting sun energy. Since altitudes of 12 to $17 \mathrm{~km}$ are not usable because of high speed jetstream winds, usually an altitude of $20 \mathrm{~km}$ is targeted for flying. Even though this leads to large, fragile airframe designs. For aerodynamic reasons and other environmental constraints, the alternative of low flying solar planes is only feasible for periods of low or no cloud coverage like demonstrated by AtlanicSolar in 2015 [1].

M. Laiacker, S. Wlach and M. Schwarzbach are with the Robotics and Mechatronics Center, German Aerospace Center (DLR), 82230 Wessling, Germany, \{maximilian.laiacker, sven.wlach, marc.schwarzbach\}@dlr.de.
In recent years companies like Google and Facebook have started their own projects aiming for HALE solar planes supplying network services worldwide.

The technologies needed for the kind of HALE (high altitude long endurance) flights mentioned are manifold. All systems have to be extremely lightweight while still working under the low pressure and varying temperature. Operational aspects of high altitude BLOS flying of UAV add to the challenges. While many problems can be solved analytically or by testing in environmental simulation facilities, the final validation can only be achieved in the real environment.

Since HALE solar platforms are not (yet) available and the construction is a major effort, the research group on flying robots of the German Aerospace Center (DLR) chose an alternative approach. Since high altitude balloon research is a well-established business and facilities are also offering segregated airspace up to the desired altitudes, the airplane for short duration testing could be designed small and without the need of propulsion when lifted to $20 \mathrm{~km}$ by a balloon . While it is not possible to test the effects of long duration exposure to altitude, results on aerodynamics, control, communication and operation can be achieved by a small team using a smaller system on a tight budget.The NASA ARES program [2] used a similar technique to simulate a plane flying in Mars atmosphere.

\section{UAV SYSTEM OVERVIEW}

The UAV, shown in Figure 1, was specifically designed for this high altitude mission and has a wingspan of $3 \mathrm{~m}$ and a mass of $7.4 \mathrm{~kg}$. It is equipped with our modular autopilot system [3] but some new modules had to be developed for this mission. The long range manual control system 


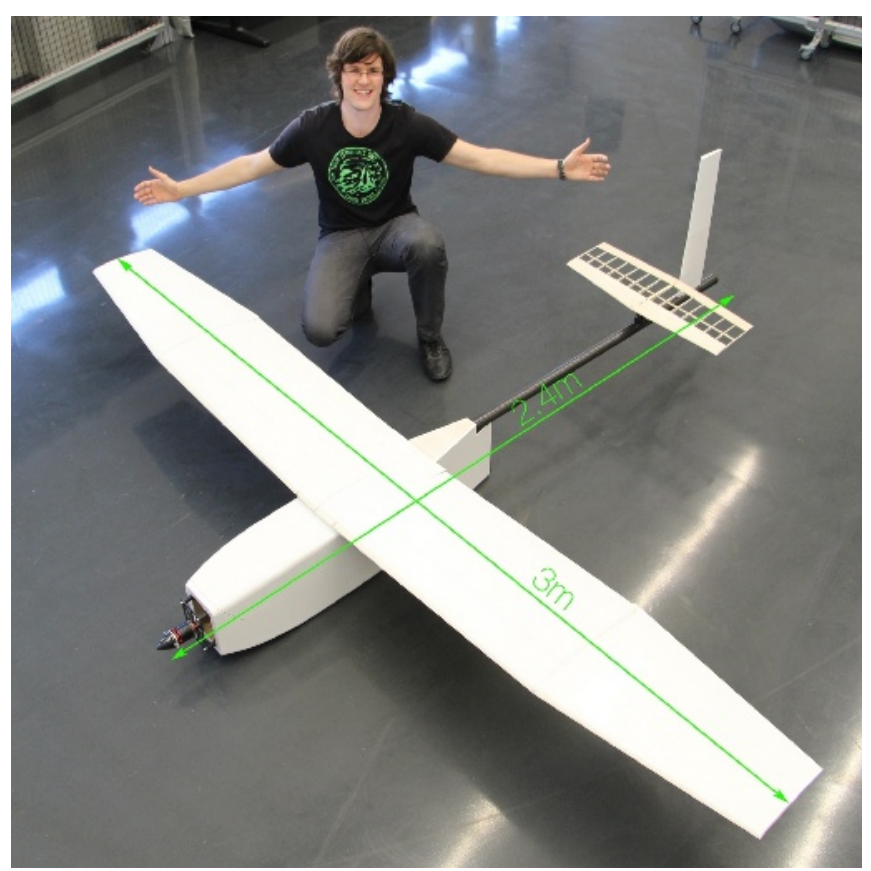

Fig. 1. The HABLEG UAV designed for high altitude missions

is also installed that allows manual flight using remote control but not in a redundant configuration. Different to the modular concept described in [3] to reduce weight and size of the overall system most of the electronic components have been integrated in one enclosure. When flying at high altitudes, thermal design of electronic components is also very important because of the very cold environment temperatures and low density atmosphere. More details of the thermal design and testing of the autopilot system are presented in [4].

The central component of the autopilot is a PowerPC based flight control computer (FCC) that runs the real time operating system QNX. The sensors and actuators are connected via serial RS232 interface to the FCC. A small calibrated MEMS inertial measurement unit is used for attitude and heading estimation. Pressure and airspeed are provided by an airdata sonde. Two u-blox GPS receivers are installed. One is in the front and one is behind the wing inside the fuselage. Two spatially separated and differently orientated U-Blox 6 GPS receivers are used for localization. They are voted for signal quality (number of satellites), which can greatly differ depending on orientation, due to the high latitude of the flight testing area. Beyond navigation, a valid GPS position is also critical to the flight

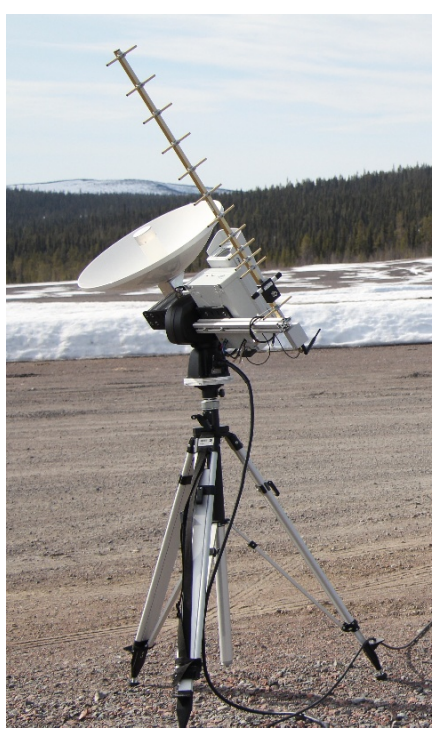

Fig. 2. The pan tilt unit with antennas used for the mission

termination logic, which in case of a telemetry and GPS failure would command the ejection of a parachute. The reception of GPS signals can easily be jammed by internal or external RF sources so using two sensors spatially separated reduces the risk that both sensors are affected. Only one sensor is used by the autopilot system and the decision which one is done automatically based on the reported number of received satellites and estimated position accuracy.

The airplane is also equipped with an analogue video downlink that is independent from the autopilot system with the exception of the power supply. This real time video downlink was added to be able to easily and directly observe the aircrafts orientation and velocity during the mission by the operator in the ground control station. Telemetry data including sensor readings, estimated state and internal states are transmitted over a low bandwidth bidirectional radio link to the ground station and displayed to the operator. The operator can also change parameters and issue commands to the UAV during the mission using this radio link.

The video downlink is using the S-Band at $2.3 \mathrm{GHz}$ which is also used for telemetry on sounding rockets that are also launched at the ESRANGE [5] test area where the mission was planned. Using this frequency allowed the usage of existing high gain tracking antenna and receiver infrastructure at the site. For the telemetry data 
radio link a dual frequency redundant system is used. The frequencies used are in the $2.4 \mathrm{GHz}$ and $868 \mathrm{MHz}$ ISM band. Using the ISM bands instead of restricted frequencies allows to use widely available commercial components and to test them without special permits before the high altitude mission. On the ground a pan-tilt unit (PTU) is used to point high gain directional antennas towards the UAV during flight. The $21 \mathrm{db}$ parabolic $2.4 \mathrm{GHz}$ and $13 \mathrm{db}$ yagi $868 \mathrm{MHz}$ antenna and PTU are shown in Figure 2. On the UAV side low gain omnidirectional antennas are used. The PTU is controlled based on the received UAV position and PTU position. During the mission there was always a working communication link to the UAV.

The ground control station is set up to accommodate three operators. The manual control operator has a remote control transmitter and a screen showing the analogue video image. The manual control operator can switch to manual mode any time if there is a working radio link and take over manual control, for example in case the autopilot control system fails. The tele command operator can send commands and parameter changes to the autopilot control system. The tele command operator also observes the video downlink, artificial horizon and position of the UAV on a map. The third operator is the flight test engineer and observes all telemetry data and coordinates the mission.

\section{MISSION SIMULATION}

To execute the mission automatically and fulfill all safety regulations, complex control software had to be implemented and tested. To test the control system a partial hardware in the loop setup was implemented. In this setup the sensor data is generated by a software module that runs on the FCC instead of the sensor drivers. This is easily done since a shared memory based middleware is used. During normal operation, several software driver processes read and interpret the data coming from the connected sensors and provide these data using the middleware to the other processes of the autopilot system. During a partial hardware in the loop setup the driver processes are stopped and simulated driver data is provided by the simulation model. The commands for the actuators provided by the control processes are read by the software drivers that communicate with the real actuators and the simulation model. The advantages of this simulation setup compared to pure software in the loop are that the motions of the actuators can be observed and the telemetry and command interface to the system is the same than during a real flight experiment. Since using this kind of simulation the human operator is part of the system the operator is also tested and trained. This operator training is very important for a successful mission since in a critical situation decisions and actions have to be executed fast. Mission checklists have also been prepared and refined during these simulations.

The simulation model supports all phases of the mission including the ascent when the UAV is tethered to the balloon. Wind influences can also be simulated to create a realistic trajectory during ascent and the resulting headwind during the glide phase. The simulation is run at real time so that the operators know what to expect during the real mission. The simulation model also supports some failure modes like a lost GPS signal. Communication failures can be simulated by simply turning of the radio modem or removing the antennas. The mission phases can be summarized as follows:

- Ascent on balloon

- Launch and drop

- Transition to horizontal flight

- High altitude flight experiments

- Return to landing position

- Landing

In Figure 3 data from a simulated transition to horizontal flight is shown. After the horizontal flight is stabilized a course towards the landing position is commanded. In this mission the landing position is equal to the position where the UAV attached to the balloon was launched. During each phase a different mode of the control system is used and the transition between these phases must be detected automatically or activated by the software. Low altitude flight test have also been performed. For these flight test the HABLEG UAV has been equipped with an electric motor. Details about the low altitude flight tests are described in [6]. 


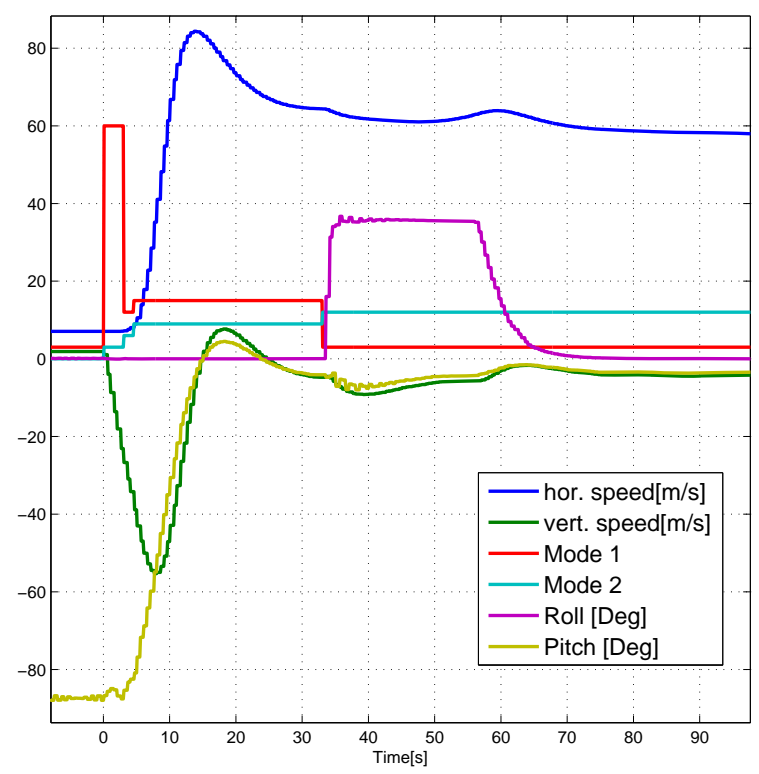

Fig. 3. Simulated balloon launch phases, velocities and angles

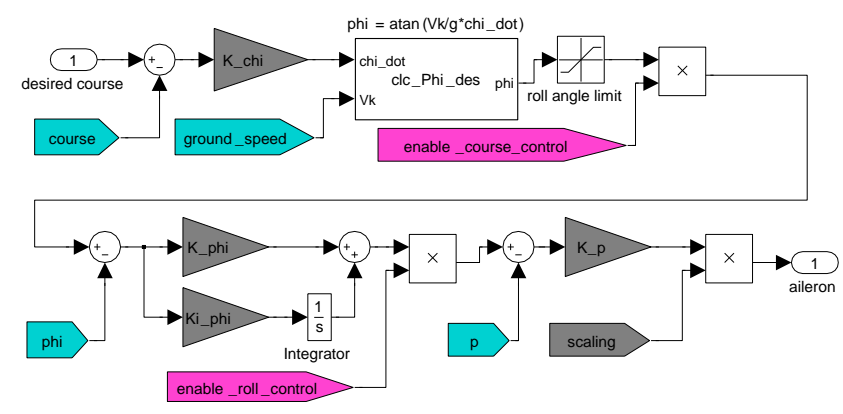

Fig. 4. Block diagram of the course controller with mode selection and gain scheduling implemented for the HABLEG mission

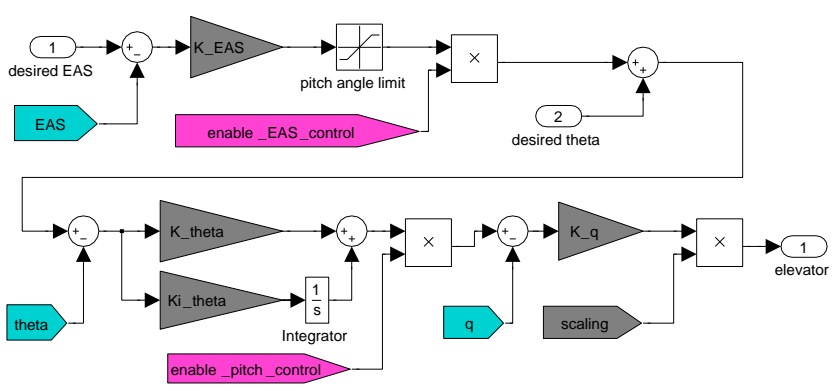

Fig. 5. Block diagram of the pitch and EAS controller with mode selection and gain scheduling implemented for the HABLEG mission

\section{Controller Design}

The controller has to support all these different flight phases in a wide range of true airspeed, altitudes and air densities. A cascaded PID controller design is used. The inner loops control the rotation rates and angles. The elevator is used to control the pitch rate $\mathrm{q}$ and the pitch angle $\theta$. The ailerons are used to control the roll rate $\mathrm{p}$ and the roll angle $\phi$. The outer loops control the equivalent airspeed EAS and the course $\chi$. In Figure 4 a block diagram of the horizontal controller for the course is shown. During the control mode "damp" the values for "enable course control" and "enable roll control" are zero. For the "roll zero" mode only "enable roll control" is set to one and for the "return to home" and waypoint mode both are set to one. The equivalent airspeed is controlled by changing the desired $\theta$ angle. The airspeed loop can be disabled by the operator and an adjustable $\theta$ angle is used. This option was added because the performance of the used airspeed sensor was not known for high altitudes. In Figure 5 the control block diagram for the pitch angle and airspeed controller is shown. During the control mode "damp" the values for "enable EAS control" and "enable pitch control" are zero. During the transition to horizontal flight the value for "enable pitch control" is slowly increased to one to make a slow transition from an almost vertical pitch angle to horizontal flight. When the airspeed control is manually enabled the value for "enable EAS control" is set to one. The desired course or heading is calculated by the navigation process or can also be entered manually by the operator. The desired $\theta$ angle is set by the operator. To adapt the controller to the different true airspeed and air density we added a gain scheduling based on the dynamic pressure for the control surfaces which resulted in stability issues which will be discussed in the next section.

\section{FLight DATA ANALYSIS}

In May 2014 a first campaign was conducted, which however didn't provide a flight opportunity due to unsuitable weather conditions. One year later, in May 2015, another - this time successful - attempt was made. The flight mission began with an overall uneventful but from the operator point of view very tense balloon ascent where 


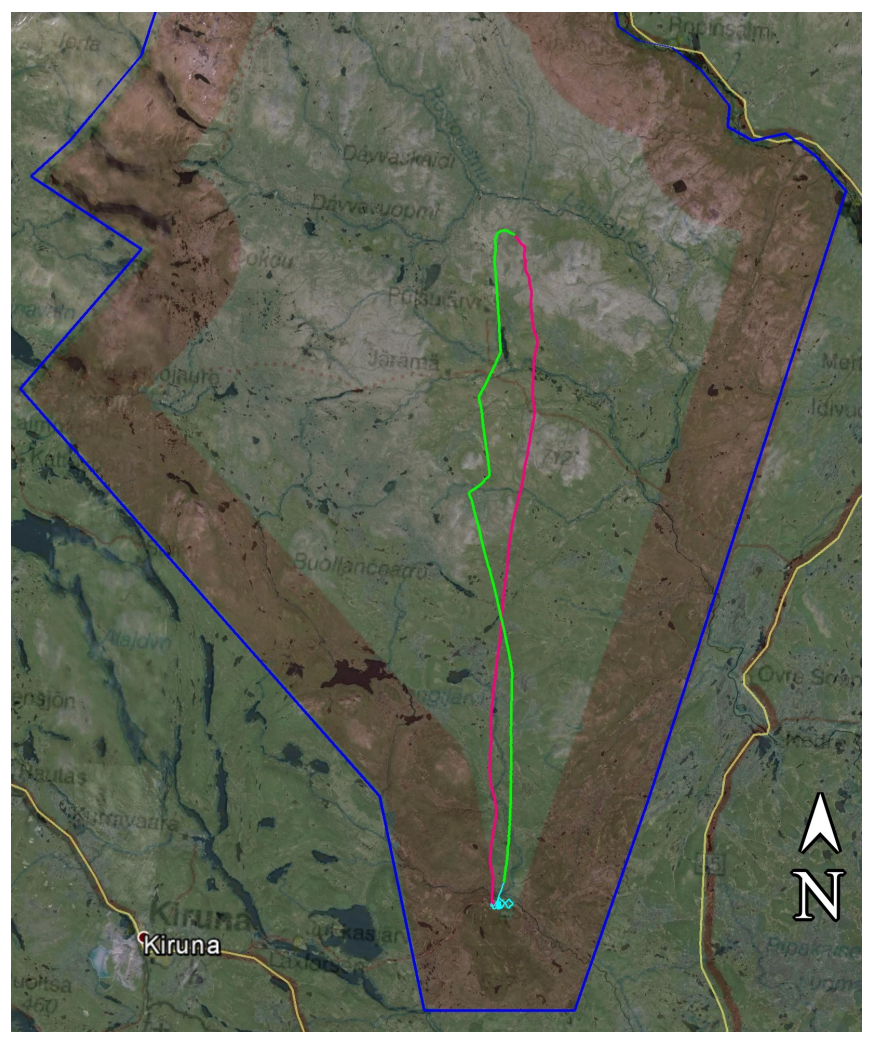

Fig. 6. Ascent (red) and descent (green) flight path during the HABLEG mission

strong winds carried the UAV to a ground distance of about $66 \mathrm{~km}$ at the maximum altitude of around $20 \mathrm{~km}$. Here the release command was issued by the ground control station. During the drop phase heavy oscillations occurred which had to be addressed by adjusting control gains by telecommand. Since this was an anticipated behavior and trained prior to the mission, the situation was handled by the ground crew within a few seconds, resulting in a safe transition to horizontal flight. After the high altitude test flight program had ended the glider entered an automatic landing pattern and finally landed close to the starting position using manual control. The mission trajectory is shown in Figure 6 and is summarized in Table I.

In the following the flight will be analyzed in more detail.

\section{A. Balloon ascent}

HABLEG was attached to the balloon at end of the tail boom so it hang vertically, nose down. The balloon train ascended very stable and started drifting north due to southerly winds. During the

\begin{tabular}{|r|r|}
\hline mission duration & 145 minutes \\
\hline ascent duration & 75 minutes \\
\hline max. altitude & $19550 \mathrm{~m}$ \\
\hline min. altitude & $330 \mathrm{~m}$ \\
\hline max. distance & $66 \mathrm{~km}$ \\
\hline total distance traveled & $169 \mathrm{~km}$ \\
\hline
\end{tabular}

TABLE I

MISSION SUMMARY

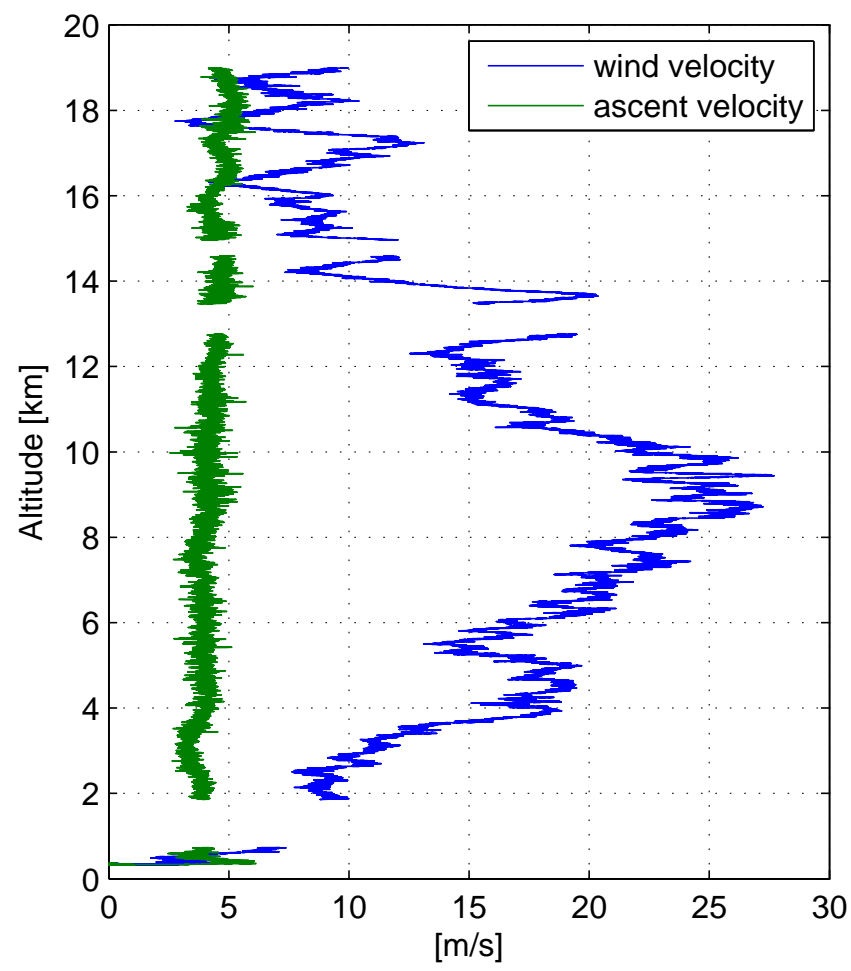

Fig. 7. Wind and ascent velocity of the balloon during ascent

ascent the autopilot was in "neutral" mode. In this mode the controller is basically switched off and all control surfaces stay in there neutral trim positions.

As the balloon got higher the horizontal ground velocity increased as the balloon entered the strongest winds around $9 \mathrm{~km}$ altitude. The wind velocity and ascent velocity profile is shown in Figure 7. An slight increase of ascent velocity with altitude can also be observed. When an altitude of $19.5 \mathrm{~km}$ was reached the release command was given.

\section{B. Transition to horizontal flight}

This is the most critical part of the mission. HABLEG has to transition from a vertical nose 


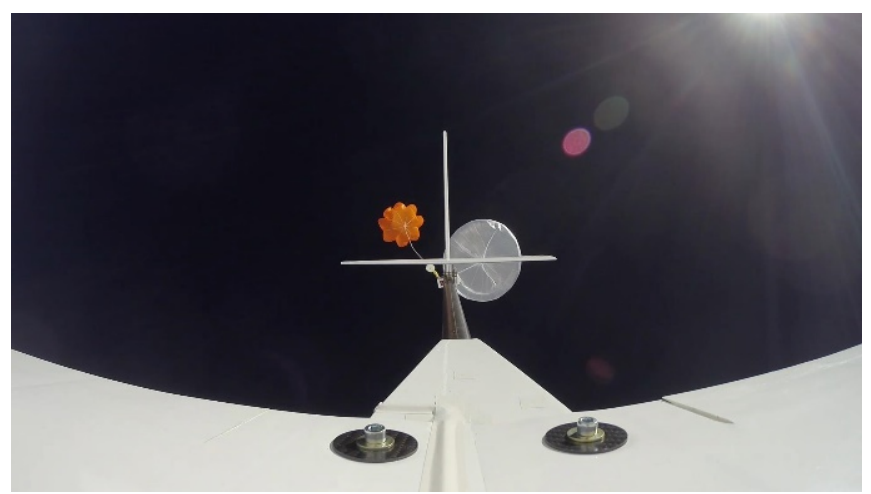

Fig. 8. Still frame from the backward facing camera shortly after releasing HABLEG from the balloon

down attitude with almost zero airspeed to horizontal flight with around $50 \frac{\mathrm{m}}{\mathrm{s}}$ true airspeed (TAS) in very thin atmosphere. During the simulation the transition happens like shown in Figure 3 where the release command was given at time zero. With $90^{\circ}$ pitch down it will pick up vertical speed at $9.81 \frac{\mathrm{m}}{\mathrm{s}^{2}}$ and the controller in "neutral" mode. A drogue chute will deploy to stabilize the drop. After a fixed amount of $3 \mathrm{~s}$ the vertical and horizontal controller are switched to "damp" mode to dampen rotational velocities. When reaching a velocity of $35 \frac{\mathrm{m}}{\mathrm{s}}$ the drogue is released and the horizontal controller controls the roll angle to zero in "roll zero" mode and the vertical controller will slowly change the pitch angle to the desired value of around $-5^{\circ}$. At $33 \mathrm{~s}$ after the release command the start sequence is finished and the horizontal controller is switch to "return to home" which will initiate a turn towards the landing position and the vertical controller is in "desired theta" mode.

During the real high altitude mission the transition to horizontal flight needed small adjustments by the operators. The release from the balloon worked perfectly. The drogue chute deployed as we could see afterwards on the video from the backward facing video camera. A still frame of this moment is shown in Figure 8. Different to the simulations the airplane started to oscillate after the "damp" mode was activated. The control gains used to dampen the rotations where too high so it had the opposite effect and lead to heavy pitch and roll oscillations which can be seen in 9 after $5 \mathrm{~s}$. These oscillations increased after the controllers are switched into the next modes "roll zero"

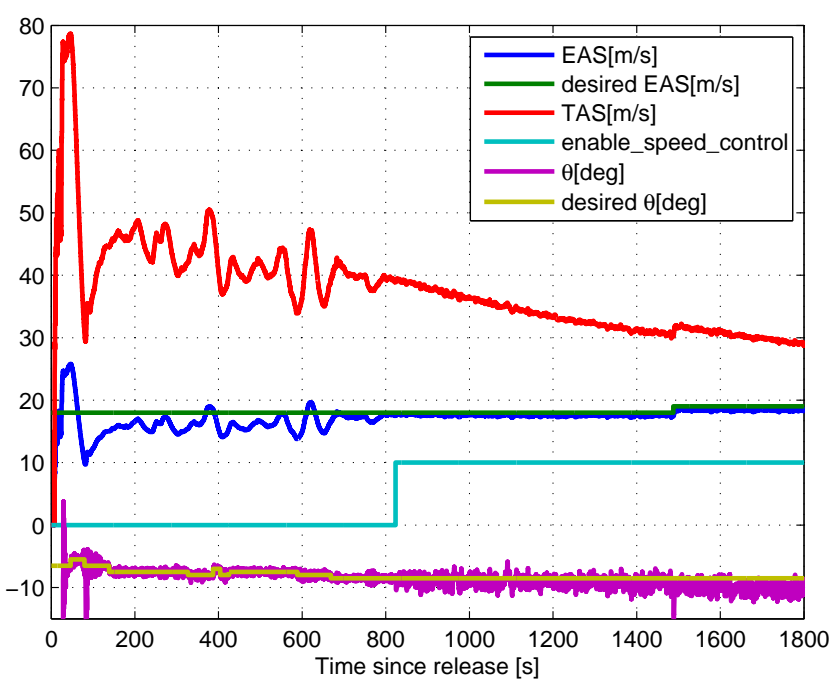

Fig. 10. Airspeed and pitch controller performance during the first half hour of the flight

and "desired theta" respectively. The operators in the ground station recognized the oscillations and started to reduce the control gain $K_{p}$ at 15 s (Fig. 11) and switched to manual control at $24 \mathrm{~s}$ (Fig. 9). When switched to manual control the controls where not used so the control surfaces stayed neutral and this immediately stopped the oscillations and rotations of the UAV as shown in Figure 9 after $25 \mathrm{~s}$. At $26 \mathrm{~s}$ the autopilot is switched on again. The horizontal controller is now controlling the roll angle to zero as planned but the vertical controller is still oscillating around the desired $\theta$ angle. The autopilot is switched off a second time at $31 \mathrm{~s}$ for $2 \mathrm{~s}$ while the control gain $K_{q}$ for the vertical controller is also reduced. At 33s the autopilot is switched on and the controller initiates a stable turn towards the landing position. The turn is finished at $55 \mathrm{~s}$ after the release command was given and HABLEG is then flying stable at around $18 \mathrm{~km}$ altitude towards the landing position still over $60 \mathrm{~km}$ away.

\section{High altitude flight}

After the turn towards the landing position was finished the airspeed started to drop. This was caused by the selected desired $\theta$ angle which was to shallow to maintain the needed airspeed. This drop in airspeed started pitch angle oscillations. The oscillation was recognized by the operators and the autopilot was switched off again for a short period of time to stop the oscillation and to 


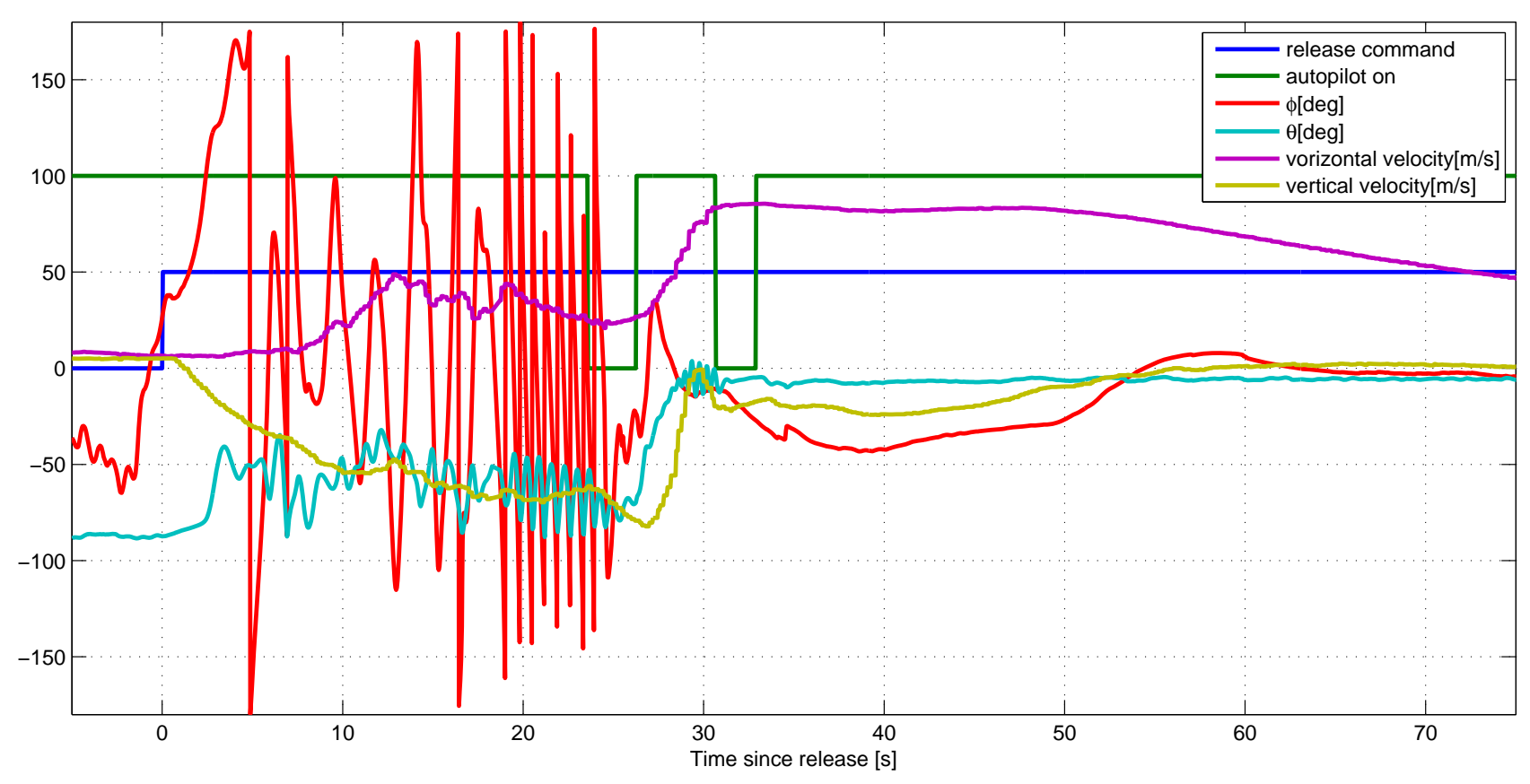

Fig. 9. Flight phases, velocities and angles shortly after release from the balloon

change gains and increase the desired $\theta$ angle at around 80s as shown in Figure 10. After these adjustments airspeed settled at around $17 \frac{\mathrm{m}}{\mathrm{s}}$ EAS and the autopilot was only switched off again shortly before the manual landing. After these control issues where solved two manual course changes where commanded to evaluate the maneuverability during high altitude flying. The course changes can be seen on the map in shown in Figure 6. These course changes with a maximum bank angle of $21^{\circ}$ were very smooth and worked as expected. About 800 s into the flight the operators verified that the airspeed sensor works reliably and the airspeed control loop was activated. The airspeed controller stabilized the airspeed very close to the set-point as shown in Figure 10 beginning 800s. The airspeed control is implemented as a $\mathrm{P}$ controller that adds an offset to the manually commanded desired $\theta$ angle so it cannot fully eliminate a steady state error. During the whole flight the operators tried to manually optimize the glide ratio by adjusting the $\theta$ pitch angle or desired airspeed as can be seen in Figure 10 at 400s and 1500s. The optimal glide ratio set point changes due to constantly changing air density and headwinds during the descent towards the landing position.
During the flight and after analyzing the recorded data a slow roll angle oscillation was detected. These oscillations originate from a quantization problem of the aileron control servos. The aileron control surfaces are quite big to provide good maneuverability at low altitudes but at higher altitudes where a higher TAS is required to generate enough lift the aileron deflections required are very small. At high speeds the aileron trim position for level flight is between two quantized servo positions which generates a slow roll oscillation because the servo will only switch position when a sufficient roll angle error is reached. Smaller control surfaces would be better suited for a high altitude UAVs with the downside of losing some maneuverability at low altitudes. The landing position was reached at about $4 \mathrm{~km}$ altitude and above the clouds. HABLEG entered an automatic landing pattern over the landing area. The final approach and landing was done under manual control to safely avoid several obstacles in the landing area. HABLEG came to a stop just $80 \mathrm{~m}$ away from where it was launched 145minutes earlier. 
D. Controller gain scheduling for high altitude flights

To adapt the controller for high altitude flight a gain scheduling of the gain $K_{p}$ and $K_{q}$ was implemented based on the ratio of the reference dynamic pressure and the current dynamic pressure.

$$
\begin{gathered}
q_{r e f}=T A S_{r e f}{ }^{2} \frac{\rho_{r e f}}{2} \\
q(T A S, \rho)=T A S^{2} \frac{\rho}{2} \\
\text { scale }_{q}(T A S, \rho)=\frac{q_{r e f}}{q(T A S, \rho)}
\end{gathered}
$$

with $T A S_{\text {ref }}=15 \frac{\mathrm{m}}{\mathrm{s}}$ and $\rho_{\text {ref }}=1.225$. This velocity scaling was used because the torque produced by a control surface deflection scales with the dynamic pressure but for a low inertia plane like a small UAV the aerodynamic damping has a big effect and results in a fast settling final rotation velocity proportional to a control surface deflection and total air speed (TAS) [7]. So scaling the control gains by dynamic pressure results in the same gains when flying at the same EAS at high altitudes but since the TAS is much higher the resulting rotation speeds are much higher, leading to an unstable controller. In Figure 11 the effective normalized control gains are shown using dynamic pressure scaling and TAS scaling with

$$
\text { scale }_{T A S}(T A S)=\frac{T A S_{r e f}}{\max \left(T A S_{r e f}, T A S\right)}
$$

where "param. 6" and "param.11" are the gain values without scaling. The "param." values are changed by the operator during the flight. At the end of the flight in lower altitudes the gain parameter are increased for better controller performance. For pressure scaling the parameters values that where changed by the operator during the flight are used to calculate the normalized gains and for TAS scaling the initial parameters are used for comparison. So using the TAS scaling would probably result in a stable controller during the critical release phase since the values are smaller than the ones used after they were changed by the operator.

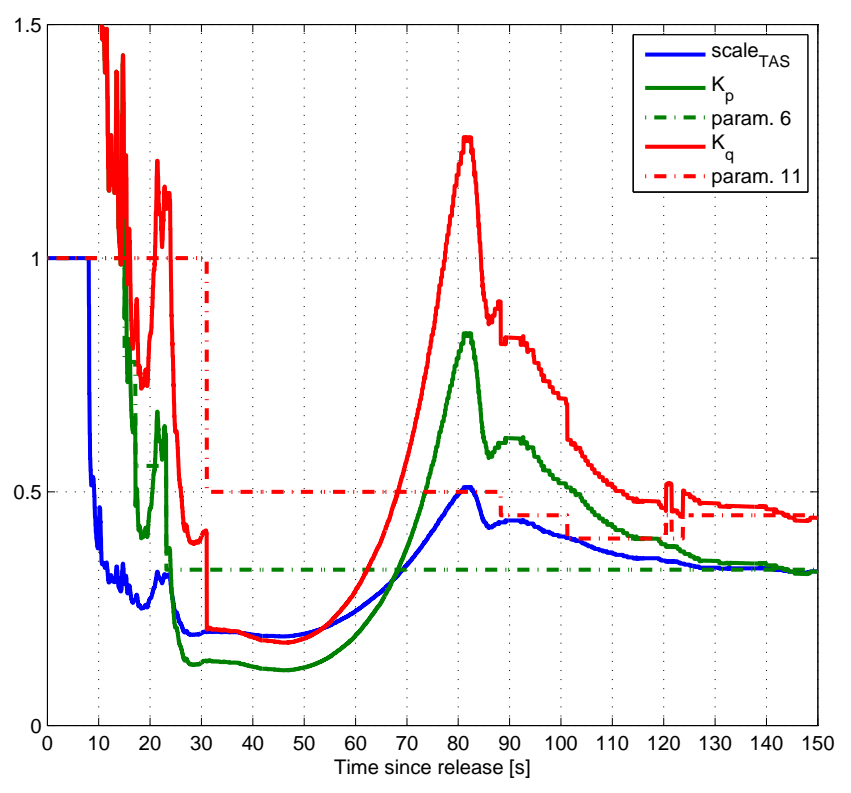

Fig. 11. Gain scheduling comparison based in TAS and dynamic pressure during the transition to horizontal flight

\section{E. GPS receiver performance}

Two u-blox GPS receivers model u-blox6 SuperSense ${ }^{\circledR}$ have been installed. Both have been configured to the "4g airborne" mode. Both receivers maintained a position fix during the hole fight. In Figure 12 the measured altitudes from both GPS receivers and the calculated pressure altitude and the number of satellites used for position calculation is shown. During the ascent when the UAVs is vertical the fluctuation in used satellites is higher because of the rotation of the yaw angle. The GPS receivers are mounted so that they point upward in normal flight so, while hanging on the balloon, they point towards the horizon. During the slow rotation during the balloon ascent the receivers have better reception from different parts of the horizon thus a different amount of satellites will be received. After the transition to stable horizontal flight the number of used satellites is more stable since now the orientation of the GPS antennas is more stable and the satellites reception is only effected by the slow motion of the satellites over the sky.

During the unstable phase of the transition to horizontal flight shortly after the release the umber of used satellites fluctuate fast and also drop by half to only 5 but this is still enough to maintain a 


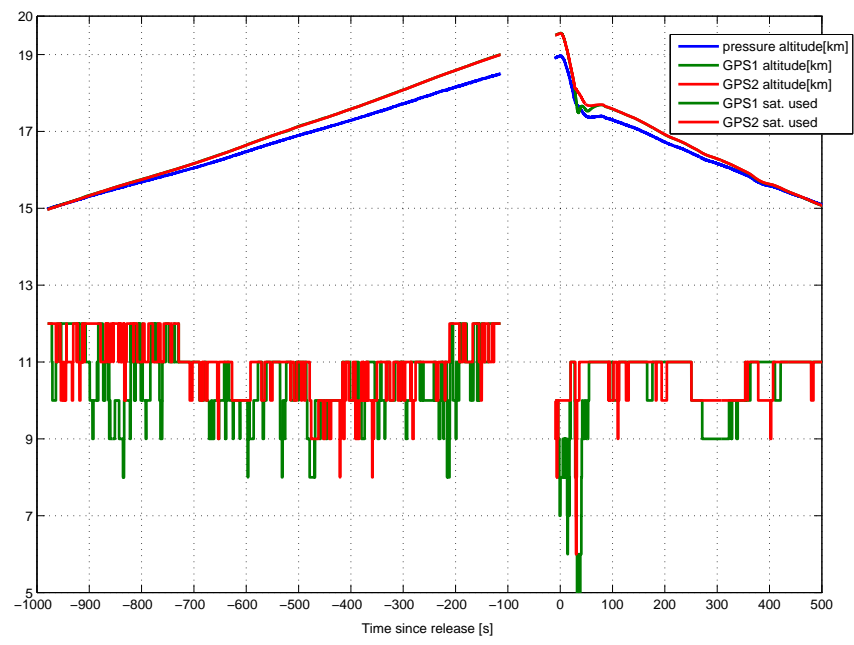

Fig. 12. GPS and pressure altitude readings during ascent and decent between 15 and $20 \mathrm{~km}$

3D position fix. During the transition from unstable drop to horizontal flight the UAV exceeded the $4 \mathrm{~g}$ limit set in the velocity model of the receivers and the reported position now differs by a large mount in the altitude. For flight control during the hole flight the GPS2 is used. By comparing the shape of the pressure altitude and GPS altitude during this phase around $50 \mathrm{~s}$ after release it can be concluded that GPS2 has calculated the correct altitude while GPS1 produced erratic measurements and the receiver voting algorithm did a good job in selecting the right receiver during the flight. The measurements of GPS1 stabilized shortly after this phase of high vertical acceleration.

The difference between the pressure altitude and GPS altitude gets bigger with altitude but there is almost no difference between the two GPS receivers. The drift of the pressure altitude can be explained by small measurement errors by the pressure sensor which result in a big altitude error in this low pressure area of the atmosphere. Between $-100 s$ and $-10 s$ the data was not recorded inside the autopilot for unknown reasons.

\section{CONCLUSIONS}

With the success of the HABLEG mission we have shown that high altitude flights are possible with this small platform. It was shown that having the partial software in the loop simulation helps a lot in creating reliable flight control software and in effective training for the operators to quickly react to problems during the mission. A more accurate aerodynamic model would have been helpful since it probably would have helped to discover the wrong approach to the control gain scheduling used for the mission. The simulation model used behaved well with the gains that result in unstable flight during the real mission. Having a real time video available also proofed to be invaluable for situational awareness. In combination with an easy to use ground station interface and intensive training, this allowed for a quick response to the encountered problem. Future work will focus on integrating the lessons learned from this mission for further high altitude missions and a possible future project for a solar powered high altitude long endurance UAV.

\section{ACKNOWLEDGMENT}

The authors thank SSC and DLR Moraba for supporting this project.

\section{REFERENCES}

[1] P. Oettershagen, A. Melzer, T. Mantel, K. Rudin, R. Lotz, D. Siebenmann, S. Leutenegger, K. Alexis, and R. Siegwart, "A solar-powered hand-launchable uav for low-altitude multi-day continuous flight," in Robotics and Automation (ICRA), 2015 IEEE International Conference on. IEEE, 2015, pp. 39863993.

[2] J.-C. Lede, R. Parks, and M. Croom, "High altitude drop testing in mars relevant conditions for the ares mars scout mission," in 2nd AIAA "Unmanned Unlimited" Conf. and Workshop, September 2003.

[3] M. Laiacker, A. Klöckner, K. Kondak, M. Schwarzbach, G. Looye, D. Sommer, and I. Kossyk, "Modular scalable system for operation and testing of uavs," in American Control Conference, 2013.

[4] S. Wlach, M. Shwarzbach, and M. Laiacker, "Dlr hableg high altitude balloon launched experimental glider," in $22 n d$ ESA PAC Symposium 2015, 2015.

[5] "Esrange Space Center SSC," http://www.sscspace.com/ esrange-space-center-3.

[6] M. Schwarzbach, S. Wlach, and M. Laiacker, "Modifying a scientific flight control system for balloon launched uav missions," in Aerospace Conference, 2015 IEEE. IEEE, 2015, pp. 1-10.

[7] H. H. Hurt, Aerodynamics for naval aviators. Office of the Chief of Naval Operations, Aviation Training Division, US Navy, 1960. 